\title{
Modified one-day etoposide and cisplatin combination for previously untreated extensive-disease small-cell lung cancer: A retrospective evaluation of 36 cases
}

\author{
SU-HEE CHO ${ }^{1}$, HYUN KUK KIM ${ }^{2}$, HANG JEA JANG ${ }^{2}$ and MIN JAE PARK ${ }^{1}$ \\ ${ }^{1}$ Division of Hematology-Oncology, Department of Internal Medicine, Pusan National University Yangsan Hospital, \\ Pusan National University School of Medicine, Yangsan; ${ }^{2}$ Division of Pulmonology, Department of Internal Medicine, \\ Inje University Haeundae Paik Hospital, Inje University College of Medicine, Busan, Republic of Korea
}

Received February 4, 2015; Accepted March 27, 2015

DOI: $10.3892 / \mathrm{mco} .2015 .556$

\begin{abstract}
The combination of etoposide and cisplatin (EP) remains one of the standard first-line treatments for extensive-disease small-cell lung cancer (ED-SCLC) We devised a one-day modified EP regimen for better tolerance and convenience by modifying the dose and schedule of conventional EP with administration over 3-5 consecutive days. The modified EP consists of two infusions of etoposide $\left(120 \mathrm{mg} / \mathrm{m}^{2}\right.$ each) and $60 \mathrm{mg} / \mathrm{m}^{2}$ of cisplatin on day 1 of a 21 -day cycle and a maximum of 6 cycles of treatment. A total of 36 consecutive ED-SCLC patients were treated with the modified $\mathrm{EP}$ as first-line therapy and retrospectively reviewed to assess the efficacy and safety of this regimen. Of the 36 patients, 24 exhibited confirmed objective tumor response (overall response rate of $66 \%$, with a complete response rate of $3 \%$ and a partial response rate of $63 \%$ ). The median overall survival (OS) was 11.8 months [95\% confidence interval (CI): 7.9-15.3] and the progression-free survival (PFS) was 7.3 months (95\% CI: 5.2-9.7). The survival estimates at 1 year were 35 and $17 \%$ for OS and PFS, respectively. The chemotherapy treatment was well tolerated, with only one case of grade 4 non-hematological adverse events, no grade 4 hematological toxicities and no treatment-related deaths. The mean relative dose intensity of etoposide and cisplatin was measured to be 94.7 and $98.5 \%$ of the planned dose, respectively. Therefore, the modified EP warrants further clinical research regarding its effectiveness, toxicity profile and convenience
\end{abstract}

Correspondence to: Dr Min Jae Park, Division of Hematology-Oncology, Department of Internal Medicine, Pusan National University Yangsan Hospital, Pusan National University School of Medicine, 20 Geumo Road, Mulgeum, Yangsan 626-770, Republic of Korea

E-mail: zoloft77@naver.com

Key words: etoposide, cisplatin, chemotherapy, small-cell lung cancer, overall survival, progression-free survival, response rate of administration. Prospective randomized clinical trials are required to determine the therapeutic role of the modified EP as first-line treatment in patients with ED-SCLC.

\section{Introduction}

Small-cell lung cancer (SCLC) is a distinct entity that accounts for $\sim 15 \%$ of all new cases of lung cancer. Approximately $60-70 \%$ of SCLC patients are diagnosed with extensive disease. Although extensive-disease-SCLC (ED-SCLC) is responsive to initial chemotherapy, it commonly progresses or relapses within months and long-term survival is poor (1).

Although various novel anticancer therapies have been developed for other types of cancer, there have been no significant advances in the therapeutic approach to ED-SCLC over the last two decades. To improve the outcome of ED-SCLC, various chemotherapeutic agents and strategies, including three-drug combinations $(2,3)$, maintenance or consolidation therapy beyond 4-6 cycles of standard chemotherapy (4), alternating or sequential combination therapies $(5,6)$ and dose-intensified regimens $(7,8)$ have been evaluated. However, none of these approaches demonstrated a significant advantage over traditional platinum-containing treatment. In a previous Japanese phase III trial (JCOG9511), the combination of irinotecan and cisplatin (IP) was found to be superior to etoposide and cisplatin (EP) for ED-SCLC (9). However, two large North American phase III trials failed to demonstrate a survival benefit of IP in comparison with EP $(10,11)$.

Etoposide in combination with a platinum agent remains one of the standard first-line treatments for ED-SCLC, with a progression-free survival (PFS) of 4-6 months and an overall survival (OS) of 8-11 months (12). During treatment with EP, patients frequently experience adverse events (AEs), mainly myelosuppression and febrile neutropenia. Grade 3-4 neutropenia occurs in $50-80 \%$ of the patients, thrombocytopenia in $10-20 \%$ and febrile neutropenia in $5-10 \%$, requiring dose modifications in a significant proportion of the patients $(10,11)$. Treatment-related AEs are associated with treatment delay, reduced quality of life, unnecessary hospitalization, increase of medical costs and, occasionally, treatment-related death. Therefore, the design of a more tolerable regimen to reduce 
AEs while maintaining acceptable efficacy is required for ED-SCLC.

Despite the use of several combination chemotherapy regimens that include etoposide, the optimal dose and schedule of etoposide administration remains unknown. An intravenous infusion of etoposide over 1-2 $\mathrm{h}$ for 3-5 consecutive days is currently widely accepted as the routine schedule in the clinical practice (13). However, several consecutive days of etoposide infusion require frequent visits to the hospital or hospitalization, causing inconvenience and additional medical costs. One-day (24-h) continuous etoposide infusion demonstrated an inferior clinical outcome compared with a daily 2-h infusion of etoposide for 5 days in SCLC (14). However, the effect of multiple 2-h infusions of etoposide within one day has not been evaluated.

In this study, we amended the dose and schedule of conventional EP and devised a modified EP, to achieve better tolerability and convenience. The aim of the present study was to retrospectively assess the efficacy and safety of this modified EP as first-line therapy administered to a consecutive series of ED-SCLC patients at a single institution.

\section{Patients and methods}

Patient eligibility. A total of 52 patients were diagnosed with SCLC at the Inje University Paik Hospital (Busan, Korea) between March, 2010 and January 2014 and ED-SCLC was confirmed in 43 of those patients. A total of 2 patients requested transfer to other hospitals for further treatment; 2 patients rejected chemotherapy treatment and received supportive care alone; and 3 patients were bedridden, with an Eastern Cooperative Oncology Group performance status (ECOG PS) of 4 and were not eligible for chemotherapy. Finally, a total of 36 consecutive patients were treated with the modified EP as first-line therapy and were included in this analysis.

This retrospective study was approved by the Local Ethics Board according to the Good Clinical Practice guidelines and applicable laws and the principles of the Declaration of Helsinki.

Treatment. The modified EP employed the lowest practicable EP dose of conventional EP regimens, including $240 \mathrm{mg} / \mathrm{m}^{2}$ etoposide and $60 \mathrm{mg} / \mathrm{m}^{2}$ cisplatin. One cycle consisted of 21 days. The sequence of chemotherapy administration for the modified EP was as follows: i) Premedication; ii) two infusions of $120 \mathrm{mg} / \mathrm{m}^{2}$ etoposide diluted in $1,000 \mathrm{ml}$ of $0.9 \% \mathrm{NaCl}$ and each administered over $120 \mathrm{~min}$; iii) infusion of $60 \mathrm{mg} / \mathrm{m}^{2}$ cisplatin over $60 \mathrm{~min}$. The patients received the modified EP as described above for a maximum of 6 cycles, unless disease progression or unacceptable toxicity occurred. Subsequent cycles of chemotherapy were permitted only if the absolute neutrophil count was $\geq 1.5 \times 10^{9} / 1$, the platelet count was $\geq 100 \times 10^{9} / 1$, the estimated glomerular filtration rate was $>60 \mathrm{ml} / \mathrm{min} / 1.73 \mathrm{~m}^{2}$ and other treatment-related non-hematological toxicities (excluding alopecia) had resolved to $\leq$ grade 1 . Treatment delay and dose modifications of EP complied with the general rules according to the grade and duration of hematological and/or non-hematological toxicities. The available modified dose level of EP was 180 and $45 \mathrm{mg} / \mathrm{m}^{2}$ for etoposide and cisplatin, respectively ( $75 \%$ of the initial target dose of the modified EP regimen). Treatment administration was recorded and relative dose intensity (RDI) (in $\mathrm{mg} / \mathrm{m}^{2} /$ week) was calculated as the total dose/body surface area divided by the number of weeks between treatment initiation and the first day of the last treatment plus 3 weeks. Granulocyte colony-stimulating factor was prescribed for patients with neutropenia under the discretion of the treating physician, but prophylactic use was not allowed.

Assessment of the response and AEs. The following tests were undertaken within 4 weeks of chemotherapy initiation: A complete history and physical examination, electrocardiogram, chest X-ray, computed tomography (CT) of the chest and upper abdomen, magnetic resonance imaging of the brain, bone scan and positron emission tomography. The response was assessed with CT scans every 2 cycles of therapy, according to the Response Evaluation Criteria in Solid Tumors (RECIST), version 1.1 (15). The severity of the AEs was graded according to the Common Terminology Criteria for Adverse Events, version 4.0 (16). The patients received the modified EP for a maximum of 6 cycles, unless disease progression or unacceptable toxicity occurred. The toxicities were assessed immediately prior to each treatment cycle and graded.

Statistical analysis. PFS was defined as the time between treatment initiation and disease progression, death, or last known follow-up (whichever occurred first). OS was defined as the interval between treatment initiation and death or last follow-up. PFS and OS were considered to have been censored at the last follow-up visit if the event had not occurred. Response rate (RR) was calculated according to RECIST 1.1. Kaplan-Meier curves were used to describe OS and PFS. Data analyses were conducted using Statistical Analysis Systems software, version 9.1 (SAS Institute Inc. Cary, NC).

\section{Results}

Patient characteristics. A total of 36 patients were included in this analysis. The baseline characteristics at diagnosis are summarized in Table I. The median age of patients was 66 years (range, $43-81$ years) and $25(70 \%)$ of the patients were men. The ECOG PS was $0-1$ in 21 (58\%), 2 in $9(25 \%)$ and 3 in $6(17 \%)$ patients.

Treatment administration. The median number of chemotherapy cycles administered was 6 (range, 2-6) and 22 (61\%) patients completed all 6 cycles. Failure to complete the 6 scheduled cycles was due to progression during treatment $(n=13,36 \%)$ and patient withdrawal $(n=1,3 \%)$. AE-related treatment delays occurred in 7 cycles in 6 patients out of 170 cycles. Dose reduction of etoposide was required in 3 patients ( $8 \%$ ) due to neutropenia and a $>25 \%$ dose reduction of etoposide $\left(180 \mathrm{mg} / \mathrm{m}^{2}\right)$ was not necessary. No dose modification of cisplatin was required. The mean RDI of etoposide and cisplatin was 94.7 and $98.5 \%$ of the planned dose, respectively.

Treatment efficacy. Of the 36 patients, 35 were evaluable for tumor response. A total of 24 patients exhibited a confirmed objective tumor response [overall response rate of $66 \%$, with a complete response rate of $3 \%$ and a partial response (PR) rate 
Table I. Baseline characteristics of the patients $(n=36)$.

\begin{tabular}{lc}
\hline Characteristics & No. of patients $(\%)$ \\
\hline Age, years & \\
Median (range) & $66(43-81)$ \\
Gender & \\
Male & $25(70.0)$ \\
Female & $11(30.0)$ \\
ECOG PS & \\
0 & $2(5.0)$ \\
1 & $19(53.0)$ \\
2 & $9(25.0)$ \\
3 & $6(17.0)$ \\
Metastatic sites & \\
Adrenal gland & $5(14.0)$ \\
Bone & $15(42.0)$ \\
Brain & $6(17.0)$ \\
Contralateral lung & $9(25.0)$ \\
Liver & $11(30.0)$ \\
Malignant pleural effusion & $14(39.0)$ \\
\hline
\end{tabular}

ECOG PS, Eastern Cooperative Oncology Group performance status.

Table II. Response to treatment $(\mathrm{n}=35)^{\mathrm{a}}$.

\begin{tabular}{lc}
\hline Type of response & No. of patients $(\%)$ \\
\hline Complete response & $1(3.0)$ \\
Partial response & $22(63.0)$ \\
Stable disease & $5(14.0)$ \\
Progressive disease & $7(20.0)$ \\
\hline
\end{tabular}

${ }^{\mathrm{a} O n e}$ patient was not assessable regarding tumor response.

of 63\%]. Five (14\%) and 7 patients (20\%) presented with stable disease (SD) and progrssive disease (PD), respectively. One patient was excluded from tumor assessment as he rejected further treatment during cycle 3 and response confirmation by CT was not available (Table II). All 36 patients were assessable for survival analysis. The median follow-up at the time of the analysis was 30.1 months. The median OS was 11.8 months [95\% confidence interval (CI): 7.9-15.3] and the PFS was 7.3 months (95\% CI: 5.2-9.7). The survival estimates at 1 year were 35 and $17 \%$ for OS and PFS, respectively (Fig. 1).

Treatment-related toxicity. All 36 patients were evaluable for safety. Treatment-related toxicities are summarized in Table III. In general, chemotherapy was well tolerated and the majority of the AEs were grade 1 or 2 . Grade 3 neutropenia and anemia were reported in 6 and $8 \%$ of the patients, respectively. Grade 4 hematological toxicities and febrile neutropenia were not observed during this treatment. Non-hematological toxicities were also tolerable. There were 2 cases of grade 3 and 1 of grade 4 non-hematological AEs during treatment.
Table III. Hematological and non-hematological adverse events.

\begin{tabular}{lcccc}
\hline & \multicolumn{4}{c}{ Grade, no. $(\%)$} \\
\cline { 2 - 5 } Adverse events $^{\mathrm{a}}$ & 1 & 2 & 3 & 4 \\
\hline Leukopenia & $9(25.0)$ & $4(11.0)$ & $2(6.0)$ & $0(0.0)$ \\
Neutropenia & $6(17.0)$ & $4(11.0)$ & $2(6.0)$ & $0(0.0)$ \\
Anemia & $16(44.0)$ & $9(25.0)$ & $3(8.0)$ & $0(0.0)$ \\
TCP & $2(6.0)$ & $0(0.0)$ & $0(0.0)$ & $0(0.0)$ \\
FN & $0(0.0)$ & $0(0.0)$ & $0(0.0)$ & $0(0.0)$ \\
Anorexia & $11(31.0)$ & $2(9.0)$ & $0(0.0)$ & $0(0.0)$ \\
Nausea & $4(17.0)$ & $3(8.0)$ & $0(0.0)$ & $0(0.0)$ \\
Vomiting & $3(8.0)$ & $1(3.0)$ & $0(0.0)$ & $0(0.0)$ \\
Diarrhea & $2(6.0)$ & $1(4.0)$ & $0(0.0)$ & $0(0.0)$ \\
Mucositis & $3(8.0)$ & $0(0.0)$ & $0(0.0)$ & $0(0.0)$ \\
Fatigue & $9(25.0)$ & $2(6.0)$ & $0(0.0)$ & $0(0.0)$ \\
Dyspnea & $5(14.0)$ & $5(14.0)$ & $1(3.0)$ & $0(0.0)$ \\
Weight loss & $3(8.0)$ & $0(0.0)$ & $0(0.0)$ & $0(0.0)$ \\
AKI & $3(8.0)$ & $0(0.0)$ & $0(0.0)$ & $0(0.0)$ \\
Infection & $0(0.0)$ & $0(0.0)$ & $1(3.0)$ & $1(3.0)$ \\
\hline
\end{tabular}

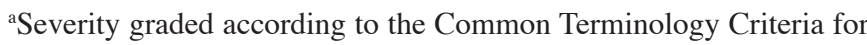
Adverse Events, version 4.0. FN, febrile neutropenia; TCP, thrombocytopenia; AKI, acute kidney injury.

The single case of grade $4 \mathrm{AE}$ was pneumonia without neutropenia occurring during the first cycle of treatment, requiring hospitalization and administration of antibiotics. The patient recovered from the pneumonia and completed 6 cycles of treatment. There was no reported treatment-related mortality.

Salvage treatment after progression. Second-line chemotherapy was administered to 25 patients, 7 (28\%) of whom had sensitive SCLC (recurring $>3$ months after completion of the initial therapy), whereas $18(72 \%)$ had resistant or refractory SCLC (progressing during chemotherapy or recurring within 3 months after completion of initial therapy). A total of 5 patients (20\%) achieved a PR, $6(24 \%)$ had SD and 14 (56\%) had PD. Third-line chemotherapy was administered to 15 patients and fourth-line chemotherapy to 6 patients (data not shown).

\section{Discussion}

This retrospective analysis assessed the efficacy and safety of the modified EP combination chemotherapy to estimate its advantages compared with conventional EP as first-line treatment in patients with ED-SCLC. The modified EP regimen of the present study applied the lowest practicable EP dose of conventional EP regimens to improve tolerance. The administration of etoposide was performed by multiple infusions within one day for better convenience, instead of conventional consecutive infusions over 3-5 days. The length of time for the completion of the treatment was $\sim 6 \mathrm{~h}$. A total of 36 patients with ED-SCLC were included in this study and the data suggested that the modified EP appeared to exhibit favorable tolerability and acceptable efficacy. The regimen achieved an overall RR 

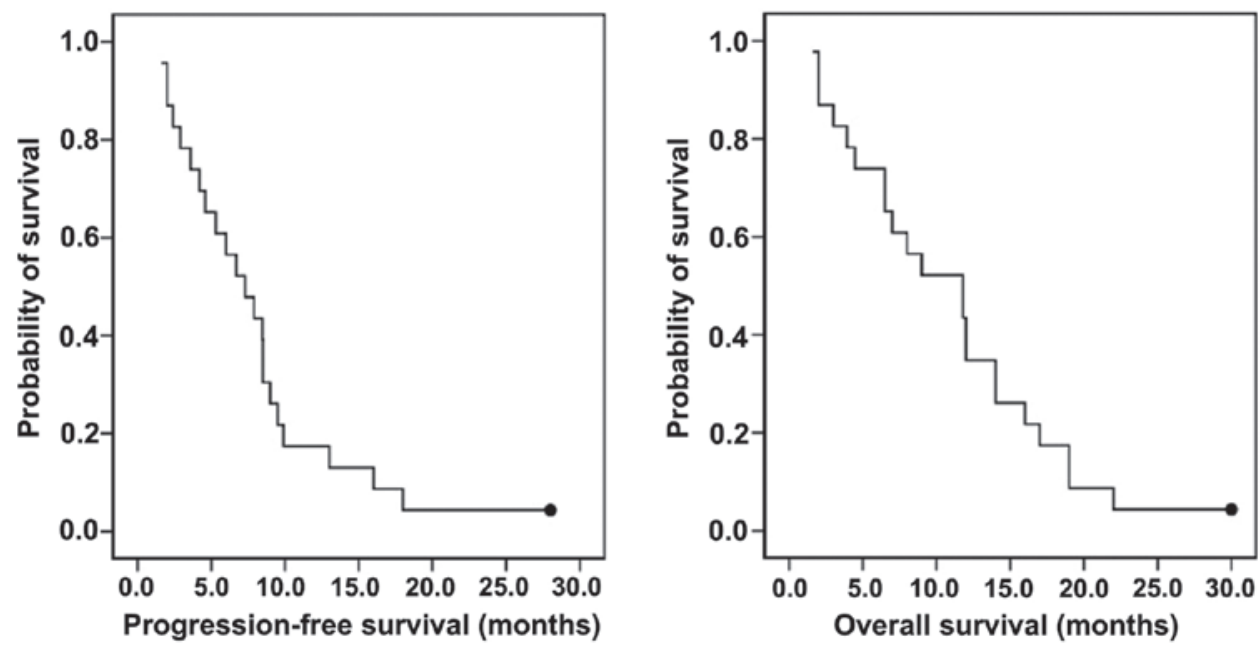

Figure 1. Kaplan-Meier plots for progression-free survival (PFS) and overall survival (OS). The median OS was 11.8 months [95\% confindence interval (CI): 7.9-15.3] and the PFS was 7.3 months (95\% CI: 5.2-9.7). The survival estimates at 1 year were 35 and $17 \%$ for OS and PFS, respectively.

of $66 \%$, with a median PFS of 7.3 months and a median OS of 11.8 months. The chemotherapy treatment was well tolerated, with only 1 case of grade 4 non-hematological AEs, no grade 4 hematological toxicities and no treatment-related deaths. The mean RDI of etoposide and cisplatin was measured to be 94.7 and $98.5 \%$ of the planned dose, respectively, indicating that the treatment was conducted without frequent dose modifications or treatment delays.

The OS of patients with ED-SCLC enrolled in phase III trials has not improved significantly over the last few years (17). Recent phase III trials investigating ED-SCLC (3,10,11,18-20) revealed a median OS of $\sim 9-11$ months, regardless of the treatment options. Lara et al (11) reported a pivotal trial including 651 patients comparing the efficacy of EP and IP as first-line treatment for patients with ED-SCLC. A total of 327 patients were assigned to the EP arm, exhibiting a median PFS of 5.2 months and a median OS of 9.1 months. Fink et al (18) evaluated the efficacy of EP and topotecan/cisplatin as first-line treatment for patients with ED-SCLC. The EP arm included 345 patients with a median time-to-progression of 5.7 months and a median OS of 9.5 months. Patients treated with the modified EP regimen exhibited a median PFS of 7.3 months and a median OS of 11.8 months, which are within the expected range of survival time for patients with ED-SCLC. Although there are clear limitations to the present study due to its retrospective nature and small patient sample, the modified EP appeared to exhibit an acceptable efficacy, despite employing the lowest practicable dose of cisplatin and etoposide of conventional EP regimens. To the best of our knowledge, there have been no clinical data to support that higher-intensity regimens yield better outcomes in the treatment of ED-SCLC patients; they are, however, associated with higher toxicity (2-8). The formulation of a less agressive regimen may be an alternative practicable approach if the treatment is equally effective, as a more tolerable regimen may decrease the incidence of treatment-related toxicities, time of hospitalization and overall medical costs during the chemotherapy treatment.

Previous clinical trials on EP for ED-SCLC demonstrated that a significant portion of the patients experienced high-grade treatment-related toxicities, leading to treatment delays and dose modifications. In the phase III study of Fink et al (18), 37.7 and 2.7\% of the 334 EP-treated patients developed grade 4 neutropenia and febrile neutropenia, respectively. Lara et al (11) reported that grade 4 neutropenia and febrile neutropenia occurred in 48 and 6\%, respectively, of 324 patients who were treated with EP. The mean RDI was $78 \%$ for etoposide and $81 \%$ for cisplatin, suggesting that frequent dose reductions and treatment delays were necessary during the treatment. The retrospective design of the present study did not allow for accurate and comprehensive assessment of treatment-related toxicities during the treatment. However, AEs during EP treatment are usually hematological and associated with myelosuppression. The hematological toxicities could be reviewed and evaluated reliably, as complete blood counts were measured routinely prior to the administration of the chemotherapy and stored as laboratory medical data. In the present study, there was no report of grade 4 neutropenia, thrombocytopenia or febrile neutropenia during treatment with the modified EP, suggesting that the modified EP may be tolerable as first-line treatment for patients with ED-SCLC. Considering its low treatment-related toxicity and acceptable efficacy, modified EP may be a viable option for elderly or frail patients, in whom AEs during treatment are a major concern.

We acknowledge a number of limitations and weaknesses of this study. First, the number of patients in the analysis was limited, which may not have provided sufficient evidence to support the efficacy and safety of the modified EP regimen. Second, survival data were collected retrospectively and comparisons were made only with historical controls, although the authors treated patients consecutively during the study period to minimize selection bias. Third, the toxicity profiles may not be complete and certain major AEs may have been missed. Therefore, the present study should be considered as preliminary regarding the efficacy and safety of modified EP and our results should be interpreted with caution. Prospective trials including adequate numbers of ED-SCLC patients must be undertaken to confirm the results of this study.

To the best of our knowledge, this is the first study to assess the efficacy and safety of EP combination with multiple 
injections of etoposide in one day as first-line treatment for ED-SCLC. Our data suggest that the modified EP regimen deserves further clinical evaluation with respect to its efficacy, toxicity profile and convenience of administration. Based upon the present study, we are planning to design a prospective randomized clinical trial to evaluate the therapeutic role of the modified EP compared with that of conventional EP as first-line treatment in patients with ED-SCLC.

\section{References}

1. Hann CL and Rudin CM: Management of small-cell lung cancer: Incremental changes but hope for the future. Oncology (Williston Park) 22: 1486-1492, 2008.

2. Pujol JL, Daurès JP, Rivière A, et al: Etoposide plus cisplatin with or without the combination of 4'-epidoxorubicin plus cyclophosphamide in treatment of extensive small-cell lung cancer: A French Federation of Cancer Institutes multicenter phase III randomized study. J Natl Cancer Inst 93: 300-308, 2001.

3. Niell HB, Herndon JE II, Miller AA, et al; Cancer and Leukemia Group: Randomized phase III intergroup trial of etoposide and cisplatin with or without paclitaxel and granulocyte colony-stimulating factor in patients with extensive-stage small-cell lung cancer: Cancer and Leukemia Group B Trial 9732. J Clin Oncol 23: 3752-3759, 2005.

4. Schiller JH, Adak S, Cella D, DeVore RF III and Johnson DH: Topotecan versus observation after cisplatin plus etoposide in extensive-stage small-cell lung cancer: E7593 - a phase III trial of the Eastern Cooperative Oncology Group. J Clin Oncol 19: 2114-2122, 2001

5. Fukuoka M, Furuse K, Saijo N, Nishiwaki Y, Ikegami H, Tamura T, Shimoyama M and Suemasu K: Randomized trial of cyclophosphamide, doxorubicin and vincristine versus cisplatin and etoposide versus alternation of these regimens in small-cell lung cancer. J Natl Cancer Inst 83: 855-861, 1991.

6. Roth BJ, Johnson DH, Einhorn LH, et al: Randomized study of cyclophosphamide, doxorubicin and vincristine versus etoposide and cisplatin versus alternation of these two regimens in extensive small-cell lung cancer: A phase III trial of the Southeastern Cancer Study Group. J Clin Oncol 10: 282-291, 1992.

7. Ihde DC, Mulshine JL, Kramer BS, et al: Prospective randomized comparison of high-dose and standard-dose etoposide and cisplatin chemotherapy in patients with extensive-stage small-cell lung cancer. J Clin Oncol 12: 2022-2034, 1994.

8. Thatcher N, Girling DJ, Hopwood P, Sambrook RJ, Qian W and Stephens RJ: Improving survival without reducing quality of life in small-cell lung cancer patients by increasing the dose-intensity of chemotherapy with granulocyte colony-stimulating factor support: Results of a British Medical Research Council Multicenter Randomized Trial. Medical Research Council Lung Cancer Working Party. J Clin Oncol 18: 395-404, 2000.
9. Noda K, Nishiwaki Y, Kawahara M, et al; Japan Clinical Oncology Group: Irinotecan plus cisplatin compared with etoposide plus cisplatin for extensive small-cell lung cancer. N Engl J Med 346: 85-91, 2002.

10. Hanna N, Bunn PA Jr, Langer C, et al: Randomized phase III trial comparing irinotecan/cisplatin with etoposide/cisplatin in patients with previously untreated extensive-stage disease small-cell lung cancer. J Clin Oncol 24: 2038-2043, 2006.

11. Lara PN Jr, Natale R, Crowley J, et al: Phase III trial of irinotecan/cisplatin compared with etoposide/cisplatin in extensive-stage small-cell lung cancer: Clinical and pharmacogenomic results from SWOG S0124. J Clin Oncol 27: 2530-2535, 2009.

12. Foster NR, Qi Y, Shi Q, Krook JE, Kugler JW, Jett JR, Molina JR, Schild SE, Adjei AA and Mandrekar SJ: Tumor response and progression-free survival as potential surrogate endpoints for overall survival in extensive stage small-cell lung cancer: Findings on the basis of North Central Cancer Treatment Group trials. Cancer 117: 1262-1271, 2011.

13. Hande KR: Etoposide: Four decades of development of a topoisomerase II inhibitor. Eur J Cancer 34: 1514-1521, 1998.

14. Slevin ML, Clark PI, Joel SP, Malik S, Osborne RJ, Gregory WM, Lowe DG, Reznek RH and Wrigley PF: A randomized trial to evaluate the effect of schedule on the activity of etoposide in small-cell lung cancer. J Clin Oncol 7: 1333-1340, 1989.

15. Eisenhauer EA, Therasse P, Bogaerts J, et al: New response evaluation criteria in solid tumours: Revised RECIST guideline (version 1.1). Eur J Cancer 45: 228-247, 2009.

16. National Cancer Institute: Common Terminology Criteria for Adverse Events (CTCAE) v4.0. http://ctep.cancer.gov/protocolDevelopment/electronic_applications/ctc.htm. Accessed May 28, 2009.

17. Oze I, Hotta K, Kiura K, Ochi N, Takigawa N, Fujiwara Y, Tabata $\mathrm{M}$ and Tanimoto M: Twenty-seven years of phase III trials for patients with extensive disease small-cell lung cancer: Disappointing results. PLoS One 4: e7835, 2009.

18. Fink TH, Huber RM, Heigener DF, et al; 'Aktion Bronchialkarzinom' (ABC Study Group): Topotecan/cisplatin compared with cisplatin/etoposide as first-line treatment for patients with extensive disease small-cell lung cancer: Final results of a randomized phase III trial. J Thorac Oncol 7: 1432-1439, 2012.

19. Schmittel A, Sebastian M, Fischer von Weikersthal L, et al; Arbeitsgemeinschaft Internistische Onkologie Thoracic Oncology Study Group: A German multicenter, randomized phase III trial comparing irinotecan-carboplatin with etoposide-carboplatin as first-line therapy for extensive-disease small-cell lung cancer. Ann Oncol 22: 1798-1804, 2011.

20. de Jong WK, Groen HJM, Koolen MGJ, Biesma B, Willems LN, Kwa HB, van Bochove A, van Tinteren H and Smit EF: Phase III study of cyclophosphamide, doxorubicin, and etoposide compared with carboplatin and paclitaxel in patients with extensive disease small-cell lung cancer. Eur J Cancer43: 2345-2350, 2007. 